\title{
Students Status Analysis and Class Teaching Effect Evaluation System Based on the Analysis of Monitoring Video
}

\author{
Qingxiang Wang \\ School of Information, Qilu University of Technology, Jinan, China \\ awqx_sd@sina.com.cn
}

Keywords: Video analysis; Students status analysis; Teaching effect evaluation; Video processing; Monitoring video

\begin{abstract}
Students status analysis is important to the teaching effect evaluation and improvement. In this paper, we propose a method to acquire the status of students by analyzing the monitoring video processing. We use the HD cameras in front of the classroom to capture the real time class video and transmit it to the servers for processing. For students status analysis, we firstly extract the student area by foreground and background segmentation and then use the skin color detection and AdaBoost classifier to find the faces of students. At last pattern recognition method is used to find the student's status. The statistical results of status could be given to the teachers for improving their teaching effect.
\end{abstract}

\section{Introduction}

Teaching effect evaluation play an important role in the process of learning and teaching. Teaching effect evaluation method mainly divided into two major categories of scale and supervisors observation of classes, both of which require the participation of people and inevitability exist certain subjectivity and also tend to have individual differences. Sometimes those are difficult to make completely accepted by the evaluation objects.

At the same time, the students as the main body of teaching process, their status in the process of course teaching can directly affect the teaching effect. The class status, such as listen to the teacher carefully, sleep, play mobile phone, etc., truly reflects the learning attitude, the interest in curriculum and whether the teaching content and process is attractive. In addition to some veteran teachers, most teachers could not pay close attention to students' class status at any time (especially the number of the larger course) and improve their teaching process according to the status of the students while they focus on teaching professional content. Teaching department also haven't the manpower to track teaching effect in all the lessons. For those reasons, there objectively requires a monitoring tools of auxiliary teaching.

In this paper, we proposed a students status analysis and class teaching effect evaluation system based on the analysis of monitoring video. Machine recognition method is used to monitor students listening states of classroom teaching and the system can automatically track the students' status in real-time. Classroom teaching process evaluation and feedback could be given to the teachers by analyzing the automatic tracked states of students listening based on the monitoring video.

In the second section, we review the related work of teaching effect evaluation and video processing method. In the third section, we will introduce our work on students status analysis and class teaching effect evaluation system for improving the teaching effect and the last section is the conclusions and future work.

\section{Related Work}

In the teaching effect evaluation theory research, Bloom divides the teaching evaluation into three parts: diagnostic evaluation, formative evaluation and summative evaluation according to the time of evaluation in the teaching implementation and the different role of evaluation [1]. There is a research trend is to put forward implementation from the summative evaluation to the process evaluation. The social root of the formulation is consistent with summative evaluation scores as the only standard to 
measure good or bad. Its utility is obvious if the purpose is to give the teacher assessment review or rating score, which can't realize adjustment and feedback of evaluation function.

Stufflebeam proposed the CIPP model which includes context evaluation, input evaluation, process evaluation and product evaluation [2]. He considered that the education process need constantly revise and improve. The process evaluation need collecting a large number of relevant information. That demand the evaluation results to feedback to the teacher, to control the teaching process by evaluation and improve teachers' teaching plan [3].

In the teaching practice, for real-time evaluation of the effect of classroom teaching, in addition to the direct observation of teachers, asking questions and in-class test in the process of teaching. Those methods will take up the course time and are difficult to refine the classroom teaching effect of each time node. For tracking teaching process, some colleges and universities installed video monitors in the classroom, such as Havard who installed an image monitoring system to track absent information of students, the main function is to count the empty seats and gather the present number, but it had not monitored the state of the students in the process of teaching.

In the video process, with the development of computer science, image processing technology, especially in the field of computer vision, acquired a lot of research results of the person's face and gesture recognition [4] in recent years. In those super-resolution image processing of the video monitoring is one of the important direction [5] and gradually applied in the teaching, such as attendance using face [6].

\section{Students Status Analysis and Teaching Effect Evaluation}

The real-time automatic monitoring method of students listening status in the process of classroom teaching is based on image recognition. That can propose a new way for the evaluation of classroom teaching. The overall design of our system can be seen in Fig. 1.

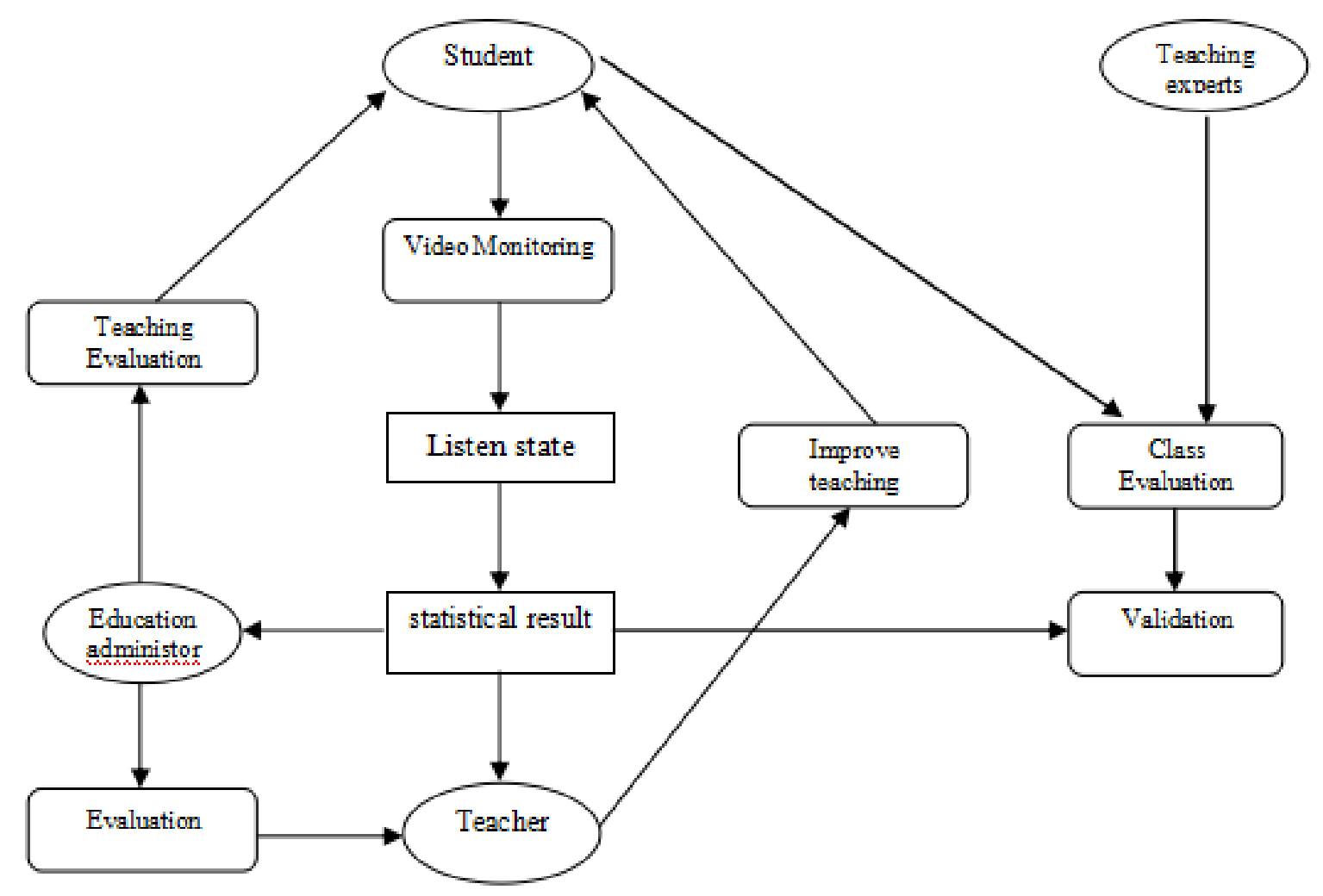

Figure 1. Overall design

Students Status Analysis. We capture the real-time video of the class by the high-definition cameras in the front of the classroom. First we segment the foreground and background combining 
with the video context from monitoring video[7] and then getting the area with student by the detection method combining skin color detection[8] and AdaBoost classifier[9] from the foreground. At last pattern recognition method, hough forests classification [10], is used to find the student's status (payable to listen, play, sleep, etc.) in these areas and analyze the results.

The monitoring cameras should be high definition cameras and place in front of the classroom and face to the student seats. It can be placed behind the platform or both sides of the board. The height is about the highest height of the student seat (for amphitheatre) or higher than 1.5 meters (for flat order big classroom). We use high-performance server to process the acquired video signal.

In this study, we assume that background basically remain unchanged in a short period of time, the difference between video frames (before the class and in class) can be used to remove the fixed part (walls, tables and chairs, etc.) and leave the motion objects (normally the students) in the classroom, and then our method could detect faces in the foreground region.

The flow of students status analysis is below:

Step 1.capture the monitoring video

Step 2. if IsClassTime

Step 3. for each frame $f$ in the video sequence

Step 4. $\quad$ segment foreground and background

selection

Step 5. use skin color detection and AdaBoost classifier to determine the preliminary

Step 6. find the student's status by hough forests classification

Step 7. analyze and save the results

Teaching Effect Evaluation: We proposed an objective evaluation method based on the students status analysis. The students statuses are more direct to reflect the quality and effect of classroom teaching than grade evaluation method, such as questionnaire survey. we analyze the recorded real-time monitoring results and the distribution characteristics of time, combining with the teaching contents and characteristics of different subjects, that can be a supplement of the existing evaluation system.

The feedback of students states in each class can be to the teacher in real-time or after class and feedback record contains a timestamp, position, the state of the students fluctuations in class and the corresponding image data, the teacher may analyze the causes of undesirable students listening states, find insufficient, which could improve the teaching plan.

After class, we count the states, calculate the total number of students of every states and the proportion of all students for a time window (e.g., 10 seconds) and then feed the fluctuation curve back to the teacher for improving the teaching effect.

\section{Conclusions and Future Work}

In this paper, we proposed a system for students status analysis and class teaching effect evaluation based on the analysis of monitoring video. The HD cameras are placed in the classroom and transmitted captured monitoring videos to the servers for processing. We use foreground and background segmentation, skin color detection and AdaBoost classifier to find the faces of students and pattern recognition method, hough forests classification, to find the student's. The statistical results of status could be given to the teachers for improving their teaching effect.

Research results can be used in the undergraduate course teaching (especially high numbers of big class teaching) effect evaluation, which can grasp the students' interest in courses by tracking state of students in the classroom in real-time. That can indirectly reflect the course content and attractive of the teaching content and teaching method. That can provide a reliable basic data source to promote the objectivity of teaching evaluation as a supplement to existing evaluation methods, at the same time, continuous student status tracking, can feed back to the teachers in time and make the teachers find easily which stages of the teaching process have existence insufficiency. Teaching departments can 
real-time track curriculum learning condition of each class and strengthen the control of teaching process automatically. That can improve the response speed of teaching feedback.

\section{Acknowledgement}

This research was supported by the Jinan star science and technology plan (No. 201406004 and No. 20120104), Qilu University of Technology education research project (No. 2016095) and partially supported by national natural science foundation of China (No 81573829) and national college students innovation and entrepreneurship training program (No.201510431010).

\section{References}

[1] B.S. Bloom, Handbook on Formative and Summative Evaluation of Student Learning, McGraw-Hill, New York, 1971.

[2] D.L. Stufflebeam, The CIPP Model for Evaluation International Handbook of Educational Evaluation, Springer Netherlands, 2001.

[3] L. J. Cronbach: Course Improvement through Evaluation, Kluwer- Nijhoff, Boston, 1983.

[4] D. M. Marsico., C. Galdi., M. Nappi., D. Riccio: FIRME: Face and Iris Recognition for Mobile Engagement, Image and Vision Computing, Vol.32 (2014) No.12, p.1161-1172.

[5] J. J. Huang, W. C. Siu: Proc. IEEE International Symposium on Circuits and Systems (Lisbon, Portugal, May 24-27, 2015), p.2161-2164.

[6] Z.S. Sabri: Research on Face Detection for Classroom Attendances Based on Skin Color Segmentation (MS., Hunan University, China 2014).

[7] O.Barnich, M.Van Droogenbroeck: ViBe: A Universal Background Subtraction Algorithm for Video Sequences, IEEE Transactions on Image Processing, Vol.20 (2011) No.6, p.1709-1724.

[8] P. Kakumanu, S. Makrogiannis, N. Bourbakis: A Survey of Skincolor Modeling and Detection Methods, Pattern Recognition, Vol.40 (2007) No.3, p.1106-1122.

[9] K. Simonyan, S. Grishin, D. Vatolin, et al: proc. IEEE International Conference on Image Processing,(San Diego, California, USA, Oct 12-15, 2008), p.349-352.

[10] J. Gall, A. Yao, N. Razavi, et al: Hough forests for object detection, tracking, and action recognition, IEEE Transactions on Pattern Analysis \& Machine Intelligence, Vol.33 (2011) No.11, p.2188-2202. 